\title{
Identifying Social Determinants of Health Leads to the Diagnoses of Scurvy in the Developed World. Case Report
}

\author{
Nikos Pappan ${ }^{1}$ (D) $\cdot$ Deeksha Sarma ${ }^{1} \cdot$ Timothy Rabe ${ }^{1} \cdot$ Rosaleen Petroccione $^{1}$ \\ Accepted: 21 February 2021 / Published online: 27 February 2021 \\ (C) The Author(s), under exclusive licence to Springer Nature Switzerland AG part of Springer Nature 2021
}

\begin{abstract}
Identifying social determinants of health can help diagnose certain nutritional deficiencies. By overcoming these barriers, we can prevent future hospitalizations and better public health. We present a unique case where a 46-year-old man presents with bilateral lower extremity swelling secondary to vitamin C deficiency. Throughout history taking, his social determinants of health were identified and he was diagnosed with scurvy from the suspected poor nutritional intake. His poor nutritional intake could have resulted from a lack of financial stability and a harsh home environment. This case is evidence that social determinants of health can directly impact a patient's well-being, and as physicians, we need to identify them to provide the most resources we can to help improve patient care. This in turn can decrease unnecessary emergency room visits and hospitalizations.
\end{abstract}

Keywords Scurvy $\cdot$ Social determinants of health $\cdot$ Vitamin C deficiency $\cdot$ Edema

\section{Background}

Scurvy is a disease that affects multiple organ systems but results from a vitamin $\mathrm{C}$ deficiency. As humans, we cannot synthesize vitamin C, and therefore, it is of utmost importance to obtain adequate levels through consumption. Scurvy affects collagen and lack of it can result in swollen gums, leg ulceration, and bleeding manifestations, most severely, cardiac failure and rhythm disturbances [1]. In developed worlds, scurvy has become a rarity due to the abundance and accessibility of goods and resources [2].

The World Health Organization defines social determinants of health as, "conditions in which people are born, grow, live, work and age. These circumstances are shaped by the distribution of money, power, and resources at global, national, and local levels [3].” Any inequality in these determinants can then affect the care or resources that an individual may have access to. Recently, the United States and even the world has been affected by the Coronavirus pandemic. This pandemic has forced states and counties to issue shutdown orders

Nikos Pappan

Nikos.Pappan@ahn.org

1 Department of Medicine, Allegheny Health Network, 320 E North Ave, Pittsburgh, PA 15212, USA which in turn has resulted in an unwanted increase in unemployment [4]. Loss of one's job can directly affect finances which have the potential to effect payment for medical bills or medical prescriptions. Even further, low income can impact payment of housing which can be associated with homelessness and mental health issues [5]. We present a unique case wherein an old age diagnosis has reappeared in the developed world secondary to inequalities in social determinants of health.

\section{Case Presentation}

A 46-year-old man with a past medical history of hypertension, tobacco use, and alcohol use disorder presented to the hospital with worsening bilateral lower extremity edema. The patient stated that over the past 2 weeks, his legs slowly became more swollen, left more so than the right. The swelling became so severe that the patient could no longer walk without assistance. The patient then noticed erythema on his right lower leg and maggot infiltration in the skin folds of his left lower leg which prompted the patient to go to the emergency room. The patient was not experiencing any fevers, shortness of breath, cough, chills, nausea, or vomiting. The patient stated that he was supposed to be on amlodipine for hypertension but had not taken it in months because the patient did not have medical insurance and could not afford any medications. 
Further social history revealed the patient was a current smoker of 1.5 packs per day for 10 years, smokes marijuana occasionally, and reportedly, drinks some beers on the weekends. The patient was working full time at the local grocery store but no longer could do so because of his lower extremity swelling. The patient lived alone and denied homelessness or harsh living conditions.

The initial workup showed that the patient was hemodynamically stable, tachycardic with a heart rate of around 130 . Physical exam was significant for bilateral lower extremity swelling with hardening of the skin as well as wound formation with maggot infiltration on the left leg (Figs. 1 and 2). A complete blood count and the complete metabolic panel were unremarkable. The patient was noted to have an elevated lactic acidosis and an elevated blood alcohol concentration. A chest $\mathrm{X}$-ray was unremarkable, and computed tomography arteriography of the lower extremities revealed severe soft tissue swelling. The patient was then admitted for suspected cellulitis of the legs and started on broad-spectrum antibiotics as well as topical permethrin to disinfect the maggots.

Additional workup was sought out to find the underlying cause of the severe lower extremity swelling because it would be unusual to have bilateral cellulitis. Workup showed normal vitamin B12, thiamine, and thyroid-stimulating hormone. HIV and hepatitis panel were negative. Also, a transthoracic echocardiogram was done which showed ejection fraction 70 $74 \%$ with no diastolic dysfunction. Right upper quadrant

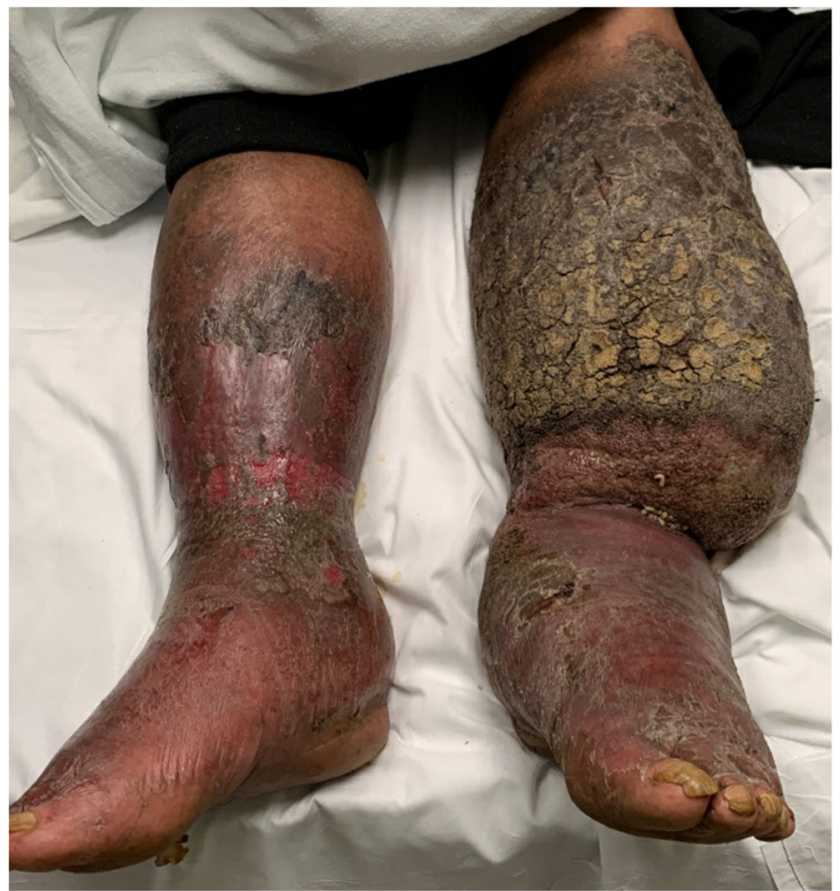

Fig. 1 Bilateral lower extremity swelling, left greater than right. Also, noted left leg chronic hardening of the skin in the calf area as well as indentation around the ankle area with few maggots noted sticking out. Right lower extremity demonstrates erythematous ulceration on the anterior shin area as well as flaking of the skin

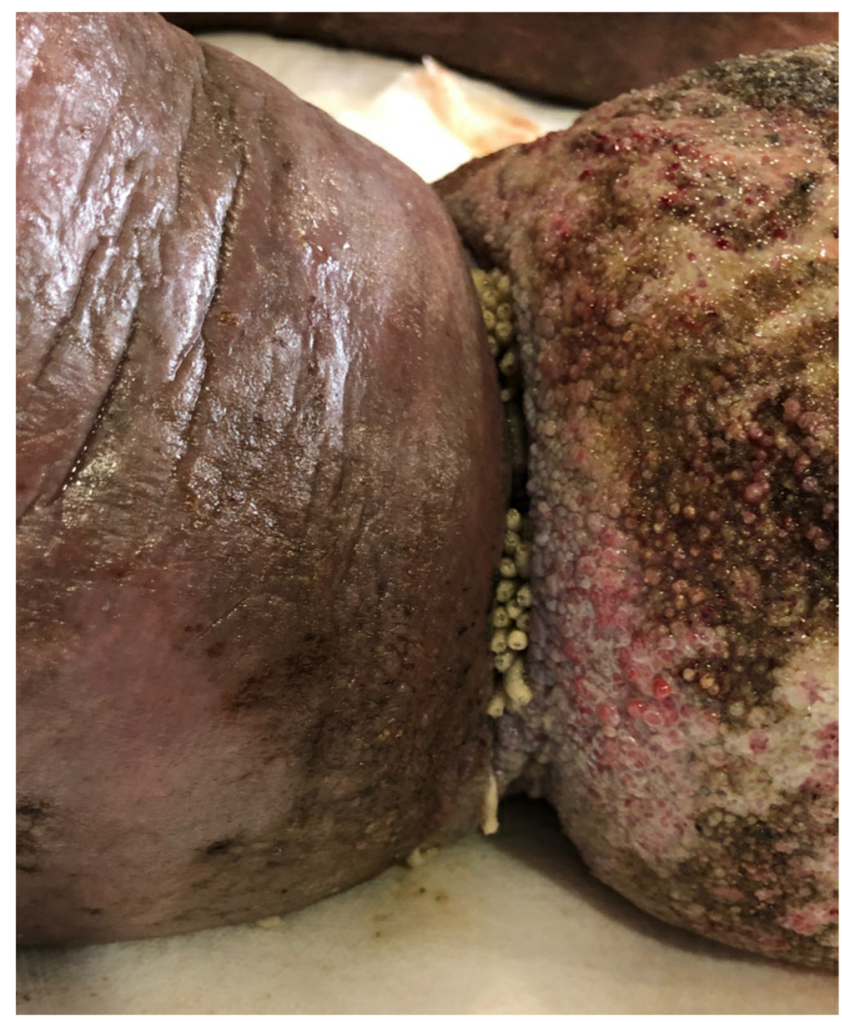

Fig. 2 Magnified picture of infoldings of skin of the left leg with noted maggot infiltration

ultrasound did not show cirrhosis. Lower extremity dopplers were unremarkable for clots. Computed tomography of the chest was unremarkable for any abnormalities. After an extensive workup with no answer, a vitamin $\mathrm{C}$ level was ordered in suspicion of nutritional deficiency which was found to be less than $0.1 \mathrm{mg} / \mathrm{dL}$ (normal value $0.4-2.0 \mathrm{mg} / \mathrm{dL}$ ). The patient was diagnosed with scurvy. Before discharge, home care was consulted for wound changes and deemed the patients home unsafe for any of their faculty to travel there. The patient was discharged with thorough instructions and adequate supplies to change his dressings as well as wound care referral to follow up with. The patient was given a month's supply of medications before he left the hospital with anticipation for PCP follow and social work help to hopefully help the patient get health insurance as well as access to food banks. Lastly, patient was prescribed vitamin $\mathrm{C}$ to wound healing.

\section{Discussion}

In this case, we present a middle-aged man who developed excessive lower extremity swelling with ulceration formation and maggot infiltration from, initially, unclear etiology. Because scurvy is seemingly rare within the developed world, more common differentials needed to be ruled out which is evident by an extensive workup. Reflecting on the case presentation, despite what the patient stated, the inability to pay 
for prescription medications and lack of medical insurance should prompt physicians to think about financial insecurities. Also, maggot infiltration of the wound could reflect poor care for oneself as well as harsh living conditions such as homelessness. Given the exclusion of more common differentials for lower extremity edema and the suspected poor nutritional status, led us to check vitamin $\mathrm{C}$ which diagnosed scurvy.

By identifying the patient's social determinants of health, we were able to diagnose scurvy. Scurvy was originally seen in sailors on boats who were at sea for months with lack of nutrition, specifically vitamin C [6]. In developed nations this is a rarity with numerous grocery stories and abundance of goods. However, with inadequate finances, a secure living environment, or inability to care for oneself diseases as scurvy can reoccur. Therefore, patients with poor social determinants of health are figuratively stranded at sea, and we as physicians are the radar to help find them and give them the resources to help them find their way back to shore. In this case, home health deemed the patients living conditions unsafe for visits; therefore, free supplies were given to the patient and referrals were made for close follow-ups to help ensure the patient did not come right back to the hospital with a severe infection. By equaling the patient's social determinants of health, we can hopefully prevent future emergency room visits and hospitalizations. In the developed world, many resources are available even if there are financial insecurities such as health food centers or food banks. It is our job as physicians to refer patients to these resources so that disease like scurvy stay old world and do not reappear.

\section{Conclusion}

This case validates the importance of identifying social determinants of health through the evaluation and diagnosis of scurvy in the developed world. As physicians, we have to assess these determinants and provide as many resources as possible for better patient care and outcomes. By equaling social determinants of health, we can hopefully reduce unnecessary emergency room visits and hospitalizations.
Code availability Not applicable.

Author's contribution Nikos Pappan drafting and revising original manuscript. Deeksha Sarma, Timothy Rabe, and Rosaleen Petroccione final revisions and approval of final manuscript.

Data Availability Not applicable.

\section{Declarations}

Ethics approval This article does not contain any research studies with human participants animals performed by any of the authors.

Consent to participate This article does not contain any research studies with human participants animals performed by any of the authors.

Consent for publication All authors give consent to publish.

Conflict of interest The authors declare that they have no conflicts of interest.

\section{References}

1. Byard RW, Maxwell-Stewart H. Scurvy-characteristic features and forensic issues. Am J Forensic Med Pathol. 2019;40(1):43-6. https:// doi.org/10.1097/PAF.0000000000000442.

2. Khalife R, Grieco A, Khamisa K, Tinmouh A, McCudden C, Saidenberg E. Scurvy, an old story in a new time: the hematologist's experience. Blood Cells Mol Dis. 2019;76:40-4. https://doi.org/10. 1016/j.bcmd.2019.01.004.

3. About social determinants of health. https://www.who.int/social determinants/sdh_definition/en/. Published September 25, 2017. Accessed July 22, 2020.

4. THE EMPLOYMENT SITUATION - Bureau of Labor Statistics. 1. https://www.bls.gov/news.release/pdf/empsit.pdf. Published 2020. Accessed July 23, 2020.

5. Forchuk C, Dickins K, Corring DJ. Social determinants of health: housing and income. Healthc Q. 2016;18:27-31. https://doi.org/10. 12927/hcq.2016.24479.

6. Carpenter KJ. The discovery of vitamin C. Ann Nutr Metab. 2012;61(3):259-64. https://doi.org/10.1159/000343121.

Publisher's Note Springer Nature remains neutral with regard to jurisdictional claims in published maps and institutional affiliations. 